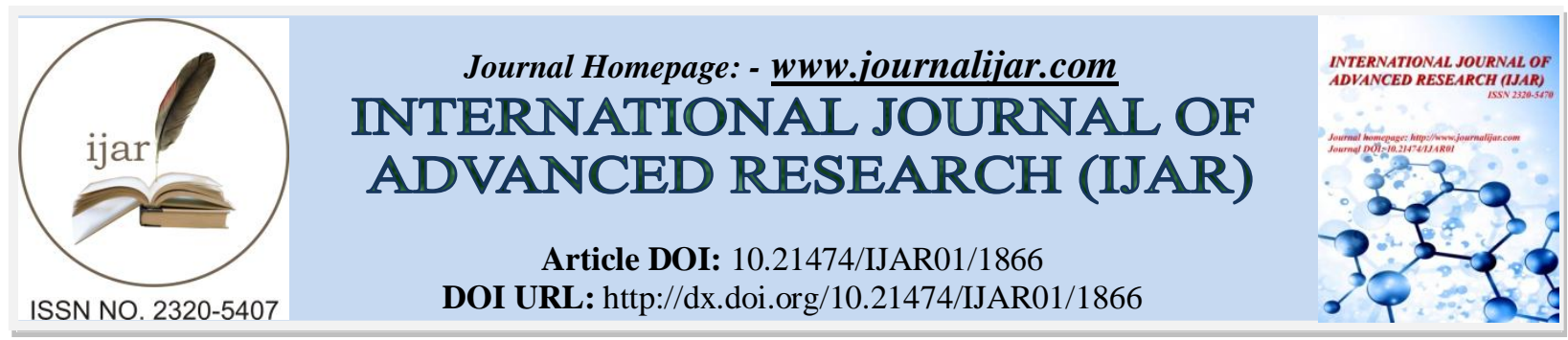

RESEARCH ARTICLE

\title{
SYNTHETIC STUDIES ON POLYIODIDES OF TRANSITION IRON(II) MEATAL TRIS-CHELATE.
}

\section{G. Pavan, Teja sree, S. Swetha ,T. Asok goud, A. Shravan kumar and M. Nagarajugoud.}

University College of Science, Saifabad, Osmania University Hyderabad - 04, india.

\section{Manuscript Info}

Manuscript History

Received: 13 August 2016

Final Accepted: 12 September 2016

Published: October 2016

Key words:-

$\left[\mathrm{Fe}(\mathrm{bpy})_{3}\right]\left(\mathrm{I}_{3}\right)_{2}, \mathrm{X}$-ray crystallography

\section{Abstract}

Synthesis and structural characterization of polyiodide mixed complex of $\mathrm{Fe}$ (II) having formula $\left[\mathrm{Fe}(\mathrm{bpy})_{3}\right]\left(\mathrm{I}_{3}\right)_{2}$, Reaction of $\mathrm{Fe}\left(\mathrm{SO}_{4}\right)_{2} \cdot 7 \mathrm{H}_{2} \mathrm{O}, 2,2$ '-bipyridine, iodine, have been described .Reaction of $\left(\mathrm{Fe}\left(\mathrm{SO}_{4}\right)_{2} \cdot 7 \mathrm{H}_{2} \mathrm{O}, 2,2\right.$ '-bipyridine,iodine $)$ produce complex in $95 \%$ yield The complex was characterized by analytical spectroscopic and molecular structure of the complex was determined by X-ray crystallography .

Copy Right, IJAR, 2016,. All rights reserved.

\section{Introduction:-}

\section{Polyiodides:-}

Polyiodides have earned much deserved attention because of their fascinating structural chemistry. The chemistry of polyiodides is vast. Up until now, a large number of polyiodide ions have been structurally characterized and mono-, di-, tri-, and tetra anions with different iodine contents have been established $\left(\mathrm{I}_{2}{ }^{-}\right.$to $\left.\mathrm{I}_{29}{ }^{3-}\right)$. ${ }^{10}$ The Lewis acid acceptor iodine and the Lewis base donors $\mathrm{I}^{-}$or $\mathrm{I}^{3-}$ can be regarded as the fundamental "building blocks" of polyiodides. The building blocks catenate easily and a large variety of polyiodides can be synthesized with various geometric features. The great variety of possible polyiodide structures is a consequence of the ability of iodine to catenate through donor-acceptor interactions combined with the influence of counter ions. These features have been rationalized and utilized in template synthesis, as well as in molecular and crystal engineering, where different cations are used to achieve special structural features. Apart from cation variation, it is also possible to substitute the polyiodide building blocks for other donor and acceptor species in order to modify the structural properties. Polyiodides are hypervalent, and many large polyiodide structures cannot be explained by simple covalent bonding models. The nature of the bonding in polyiodides has therefore been the object of much theoretical speculation. ${ }^{11}$ The stability of the polyiodide anion is largely dependent on the cation chosen. It has been found that most stable salts are formed when the cation and anion are of nearly same size. ${ }^{12}$

\section{Objectives of the present project:-}

The large majority of polyhalide ions have been crystallized as salts of organic cations (for example, aminium ions). The ability of tris-chelate complex cations (mainly dications) to generate novel polyhalide structures has been demonstrated. ${ }^{1-3}$ The present project was undertaken with the objective of preparing and structurally characterising polyiodides using tris-chelate counterions. Since analytical and crystallographic characterization could not be completed in most cases, the formulae given under the following sections are tentative. 


\section{Experimental section:-}

Materials:-

All chemicals, $\mathrm{Fe}\left(\mathrm{SO}_{4}\right)_{2} \cdot 7 \mathrm{H}_{2} \mathrm{O}, 2,2^{\prime}$-bipyridyl, Potassium Iodide, Iodine, acetonitrile and DCM were of reagent grade obtained from commercial sources and were used without further purification.

\section{Synthesis of $\left[\mathrm{Fe}(\mathrm{bpy})_{3}\right]\left(\mathrm{I}_{3}\right)_{2} \cdot \mathbf{C H}_{3} \mathbf{C N}(\mathbf{1})$ :-}

$\mathrm{Fe}\left(\mathrm{SO}_{4}\right)_{2} \cdot 7 \mathrm{H}_{2} \mathrm{O}(0.500 \mathrm{~g}, 1.79 \mathrm{mmol})$ and 2,2'-bipyridine $(1.00 \mathrm{~g}, 6.40 \mathrm{mmol})$ were dissolved in $20 \mathrm{~mL}$ of water. To this solution, iodine $(1.00 \mathrm{~g}, 3.93 \mathrm{mmol})$ dissolved in $50 \mathrm{~mL}$ dichloromethane, solid $\mathrm{KI}(0.664 \mathrm{~g}, 4.00 \mathrm{mmol})$ and dil.Sulphuric acid (1.0 M, $1.0 \mathrm{~mL}$ ) were added simultaneously and the two-phase reaction mixture was stirred for 20 minutes. The filtrate was transferred into the separating funnel and the dark red coloured organic layer was extracted with an additional amount $(30 \mathrm{~mL})$ of dichloromethane and dried over $\mathrm{Na}_{2} \mathrm{SO}_{4}$ overnight. The black crystalline precipitate formed was collected and dried. Filtered organic extracts were allowed for crystallisation at low temperature $\left(5^{\circ} \mathrm{C}\right)$. After six days square shaped black crystals were obtained and collected for SC XRD.

Yield: $2.2987 \mathrm{~g}(1.702 \mathrm{mmol}, 95 \%)$

CHN data: C, 26.23; H, 1.75; N, 6.69 .

Significant IR Absorptions $\left(\mathrm{cm}^{-1}\right): 3049.64,1608.03,1561.54,1468.55,1442.72,1303.24,1267.08,1158.60$, $1019.12,957.13,884.81,843.48,760.82,719.50,600.68$

Table 1:- Crystallographic data and structure refinement for $\left[\mathrm{Fe}(\mathrm{bpy})_{3}\right]\left(\mathrm{I}_{3}\right)_{2} \cdot \mathrm{CH}_{3} \mathrm{CN}(\mathbf{1})$

\begin{tabular}{|l|l|}
\hline & \\
\hline Formula & $\mathrm{C}_{34} \mathrm{H}_{30} \mathrm{FeI}_{6} \mathrm{~N}_{8}$ \\
\hline Formula weight & 1367.91 \\
\hline Crystal system & Orthorhombic \\
\hline$a(\AA)$ & $20.684(3)$ \\
\hline$b(\AA)$ & $10.8582(16)$ \\
\hline$c(\AA)$ & $19.124(3)$ \\
\hline$\alpha\left(^{\circ}\right)$ & 90 \\
\hline$\beta\left(^{\circ}\right)$ & 90 \\
\hline$\gamma\left({ }^{\circ}\right)$ & 90 \\
\hline$V\left(\AA^{3}\right)$ & 1367.91 \\
\hline$Z$ & 4 \\
\hline Space group & $P b c n$ \\
\hline$T(\mathrm{~K})$ & $298(2)$ \\
\hline$\rho_{\text {calcd. }}\left(\mathrm{g} \mathrm{cm}^{-3}\right)$ & 2.115 \\
\hline$\mu\left(\mathrm{mm}^{-1}\right)$ & 4.697 \\
\hline Crystal size $\left(\mathrm{mm}^{3}\right)$ & $0.22 \times 0.18 \times 0.16$ \\
\hline$\theta$ Range $\left(^{\circ}\right)$ & $1.969-28.299$ \\
\hline$h / k / l$ indices & $-27,27 /-14,14 /-25,25$ \\
\hline Reflect., collectd & 47035 \\
\hline Unique reflect., Rint & $5230,0.0420$ \\
\hline GOOF & 1.098 \\
\hline$R I[I>2(\sigma l)]$ & 0.0494 \\
\hline$w R 2[$ all data] & 0.1081 \\
\hline
\end{tabular}

\section{Physical measurement:-}

IR spectra were recorded by using KBr pellets on a Jasco 5300 FT/IR infrared spectrometer.

\section{X-ray Crystallography:-}

X-ray data was collected for compound $\mathbf{1}$ on a Bruker D8 Quest APEX CCD X-ray diffractometer, using graphitemonochromated Mo-K $\alpha$ radiation $(\lambda=0.71073 \AA)$. Data were reduced using SAINTPLUS ${ }^{30}$ and a multi-scan absorption correction using SADABS ${ }^{31}$ was performed. The structure was solved using SHELXS-97 $7^{32}$ and full 
matrix least squares refinement against $\mathrm{F}^{2}$ was carried out using SHELXL-97. All ring hydrogens were assigned on the basis of geometrical considerations and were allowed to ride upon the respective carbon atoms.

\section{Results and Discussion:-}

The complex $\mathbf{1}$ is crystallizes in the orthorhombic system, space group $P \mathrm{~b} c \mathrm{n}$, with 4 molecules in the unit cell. The structure consists of $\left[\mathrm{Fe}(\mathrm{bpy})_{3}\right]^{2+}$ cations and two $\mathrm{I}_{3}{ }^{-}$anions. Views of cation and anions are shown in Figure 1 . The crystal consists of a $\left[\mathrm{Fe}(\mathrm{bpy})_{3}\right]^{2+}$ cation on a two- fold axis and two $\mathrm{I}_{3}{ }^{-}$anions one of which sits on a two-fold axis of symmetry. The $\left[\mathrm{Fe}(\mathrm{bpy})_{3}\right]^{2+}$ cation, possesses an average $\mathrm{Fe}-\mathrm{N}$ bond distance of $1.968 \AA$ and the average $\mathrm{N}-\mathrm{Fe}-\mathrm{N}$ bond angle of $81^{\circ}$. Geometrical parameters are presented in Table 2 .
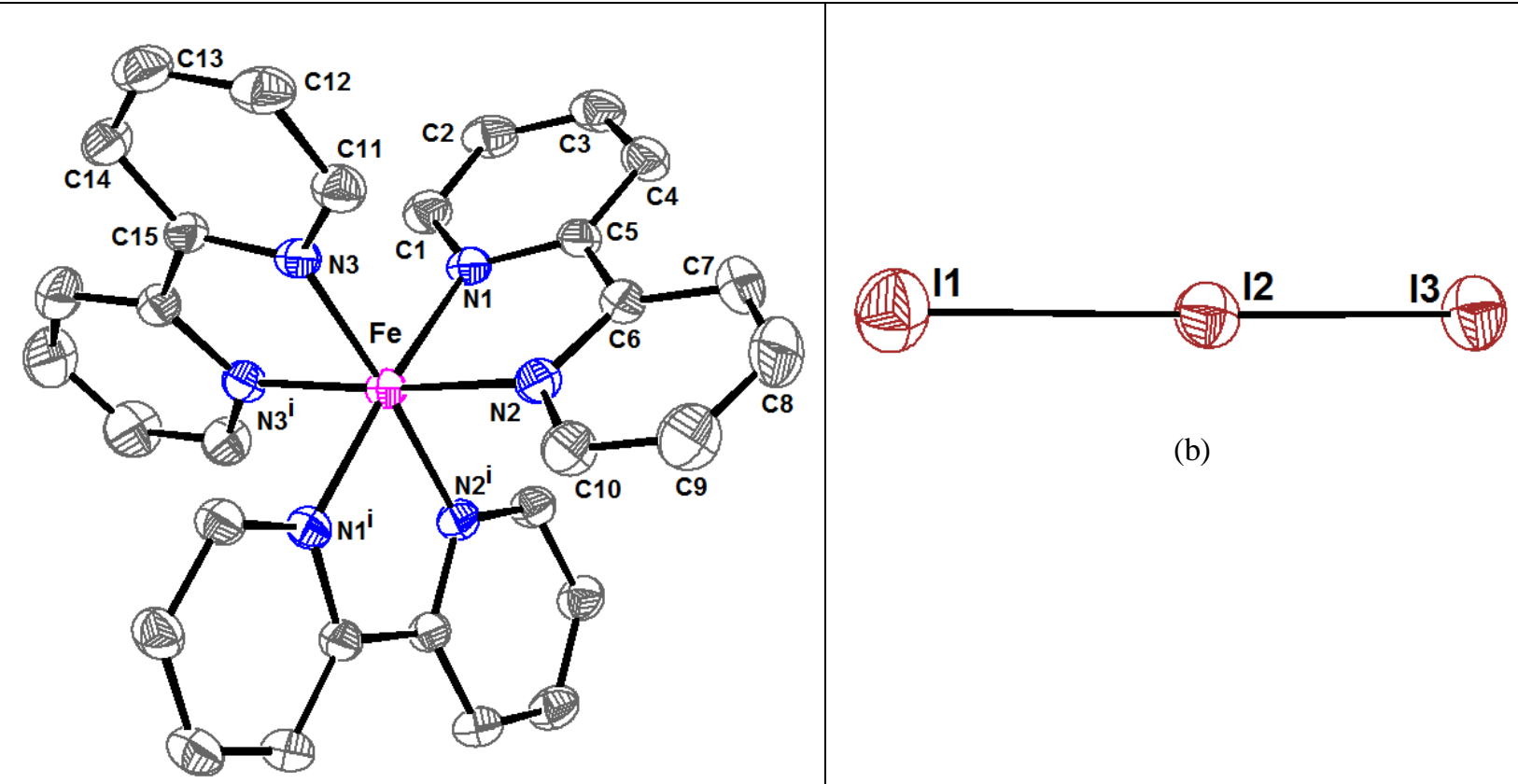

(a)

Figure. 1:- ORTEP view of $\left[\mathrm{Fe}(\mathrm{bpy})_{3}\right]\left(\mathrm{I}_{3}\right)_{2} \cdot \mathrm{CH}_{3} \mathrm{CN}$ in 1. (a) Tris chelate of $\left[\mathrm{Fe}(\mathrm{bpy})_{3}\right]^{2+}$;

(b) nearly leaner symmetrical $\mathrm{I}_{3}{ }^{-}$. Atoms are shown as $30 \%$ thermal ellipsoids. Hydrogen atoms

and lattice solvent acetonitrile molecule are omitted for clarity. (i) $=-x, y,-z+3 / 2$.

Table 2:- Selected bond lengths $[\AA]$ and bond angles $\left[{ }^{\circ}\right]$ for compound 1.

\begin{tabular}{|c|c|c|c|c|c|}
\hline $\mathrm{Fe}-\mathrm{N}(1)$ & $1.973(4)$ & $\mathrm{Fe}-\mathrm{N}(2)$ & $1.963(4)$ & $\mathrm{Fe}-\mathrm{N}(3)$ & $1.969(4)$ \\
\hline Fe-N(1)\#1 & $1.973(4)$ & $\mathrm{Fe}-\mathrm{N}(2) \# 1$ & $1.963(5)$ & $\mathrm{Fe}-\mathrm{N}(3) \# 1$ & $1.969(4)$ \\
\hline $\mathrm{I}(1)-\mathrm{I}(2)$ & $2.9186(7)$ & $\mathrm{I}(2)-\mathrm{I}(3)$ & $2.9201(7)$ & & \\
\hline $\mathrm{N}(1)-\mathrm{Fe}-\mathrm{N}(2)$ & $81.51(17)$ & $\mathrm{N}(2)-\mathrm{Fe}-\mathrm{N}(3)$ & $94.75(16)$ & $\mathrm{N}(1)-\mathrm{Fe}-\mathrm{N}(3)$ & $87.78(17)$ \\
\hline $\mathrm{N}(2)-\mathrm{Fe}-\mathrm{N}(2) \# 1$ & $89.2(2)$ & $\mathrm{N}(2)-\mathrm{Fe}-\mathrm{N}(3) \# 1$ & $175.47(16)$ & $\mathrm{N}(3)-\mathrm{Fe}-\mathrm{N}(3) \# 1$ & $81.4(2)$ \\
\hline $\mathrm{N}(2) \# 1-\mathrm{Fe}-\mathrm{N}(1)$ & $95.05(17)$ & $\mathrm{N}(2) \# 1-\mathrm{Fe}-\mathrm{N}(3) \# 1$ & $94.75(16)$ & $\mathrm{N}(3) \# 1-\mathrm{Fe}-\mathrm{N}(1)$ & $95.87(17)$ \\
\hline $\mathrm{N}(2) \# 1-\mathrm{Fe}-\mathrm{N}(1) \# 1$ & $81.51(17)$ & $\mathrm{N}(3) \# 1-\mathrm{Fe}-\mathrm{N}(1) \# 1$ & $87.78(17)$ & $\mathrm{N}(1)-\mathrm{Fe}-\mathrm{N}(1) \# 1$ & $175.2(2)$ \\
\hline $\mathrm{I}(1)-\mathrm{I}(2)-\mathrm{I}(3)$ & $177.63(2)$ & & & & \\
\hline
\end{tabular}

Redox properties of the bipyridyl derivatives of the transition metals are highly connected with their great redox capacity, which is in turn due to the branched $\pi$ system of the bipyridyls.

These complexes are nearly isostructural .Metal atoms with +2 oxidation state are oxidized to +3 by Bromine and Iodine. For metals the oxidation of $\mathrm{M}^{2+}$ to $\mathrm{M}^{3+}$ occurs due to the large reduction potential values of $\mathrm{M}^{2+} / \mathrm{M}^{3+}$ and $\mathrm{Br}_{2} / \mathrm{Br}^{-}\left(1.07 \mathrm{E}^{0}\right)$ or of $\mathrm{M}^{2+} / \mathrm{M}^{3+}$ and $\mathrm{I}_{2} / \mathrm{I}^{-}(-0.535)$. The reduction potential values for the metals are $\mathrm{Co}^{2+} / \mathrm{Co}^{3+}(-$ $\left.1.80 \mathrm{E}^{0}\right), \mathrm{Fe}^{2+} / \mathrm{Fe}^{3}\left(-0.77 \mathrm{E}^{0}\right), \mathrm{Mn}^{2+} / \mathrm{Mn}^{3+}\left(-1.51 \mathrm{E}^{0}\right)$. 
$\mathrm{M}^{2+}+2 \mathrm{Br}^{-}+3$ bpy $+9 / 2 \mathrm{Br}_{2} \rightarrow\left[\mathrm{M}(\mathrm{bpy})_{3}\right]\left(\mathrm{Br}_{3}\right) 1.5\left(\mathrm{Br}_{8}\right)_{0.25}$

$\mathrm{M}^{2+}+2 \mathrm{I}^{-}+3$ bpy $+9 / 2 \mathrm{Br}_{2} \rightarrow\left[\mathrm{M}(\mathrm{bpy})_{3}\right]\left(\mathrm{I}_{3}\right) 1.5\left(\mathrm{I}_{8}\right)_{0.25}$

\section{Conclusion:-}

The formation of unusual polyiodide anions during the synthesis of the present set of complexes shows that subtle differences in the size and shape of the cations can profoundly infuence the structure an anions. A detailed study of the synthesisied complexes could help in achieving polyhalide species with special physical properties arising from charge transfer electronic transitions and low-dimensional solid-state structures of polyhalide anions.

\section{References:-}

1. A.I. Popov, In: V. Gutmann, (Ed.), Halogen Chemistry, Academic Press, London, 1967, 1, 225.

2. A.J. Down, C.J. Adams, J.C. Bailer, H.J. Emeleaus, R. Nyholm, A.F. Trotman-Dickenson (Eds.), Comprehensive Inorganic Chemistry, Pergamon, Oxford, 1973, 3, 1476.

3. P. Coppens, J.S. Miller, (Ed.), Extended Linear Chain Compounds, Plenum, New York, 1982, 333.

4. Karpfen, A. in Halogen Bonding - Fundamentals and Applications, Springer-Verlag, 2008, 126

5. G.A. Landrum, N. Goldberg, Hoffmann, J. Chem. Soc. Dalton Trans. 1997, 3605.

6. M.C. Aragoni, M. Arca, F.A. Devillanova, A. Garau, F. Isaia, V. Lippolis, Mancini, A. Bioinorg. Chem. Appl. 2007, 2007, 46.

7. A.I. Popov, MTP International Review of Science, Inorg. Chem.Series One, 1972, 3, 53.5.

8. M.E. Easton, A. J. Ward, T. Hudson, P. Turner, A. F. Masters, T. Maschmeyer, Chem. Eur. J. $2015,21,2961$.

9. V. T. Calabrese, A. Khan, J. Phys. Chem. A 2000, 104, 1287.

10. A.J. Blake, F.A. Devillanova, R.O. Gould, L. Sheung, W. Lippolis, V. Parsons, S. Radek, C. Schroder, M. Chem. Soc. Rev. 1998, 27,

11. P. H. Svenson, L. Kloo, Chem. Rev. 2003 , $103,1649$.

12. D Ramalakshmi, Shodganga. 2011, 2, 13.

13. K. F. Tebbe, A. L. Rheingold, Elsevier, Amsterdam, 1977, 551.

14. H. Stegemann, A. Rohde, A. Reiche, A. Schnittke, H. Fullbier, Electro. chim. Acta, 1992, 37, 379.

15. G. R. Desiraju, P. S. Ho, L. Kloo, A. C. Legon, R. Marquardt, P. Metrangolo, P. Politzer, G. Resnati, K. Rissanen, Pure Appl. Chem., 2013, 85, 1711.

16. (a) T. Welton, Chem. Rev. 1999, 99, 2071. (b) K.R. Seddon, J. Chem. Technol. Biotechnol. 1997, 68, 351.

17. M. Horn, S.I. Dance, Cryst. Eng. Comm 2000, $2,53$.

18. H. Haller, M. Hog, F. Schold, H. Scherer, I. Krossing, S. Riedel, Z. Naturforsch. 2013, 68B, 1103.

19. K.N. Robertson, P.K. Bakshi, T.S. Cameron, O. Knop, Z. Anorg. Allg. Chem. 1997, 623, 104.

20. C.W. Cunningham, G.R. Burns, V. McKee, Inorg. Chim. Acta, 1990, 167, 135.

21. M. Wolff, J. Meyer and C. Feldmann, Angew. Chem., Int. Ed. 2011, 50, 4970.

22. F. B. Alhanash, N. A. Barnes, S. M. Godfrey, R. Z. Khan and R. G. Pritchard, Polyhedron, 2013, 65, 102.

23. M.E. Easton, A.J. Ward, T. Hudson, P. Turner, A.F. Masters, T. Maschmeyer, Chem. Eur. J. 2015, $21,2961$.

24. M.C. Aragoni, M. Arca, F.A. Devillanova, F. Isaia, V. Lippolis, A. Mancini, L. Pala, A. M. Z. Slawin and J. D. Woollins, Chem. Commun., 2003, 2226.

25. S. N. Bajpai, J. Chem. Eng. Data, 1981, 26, 2.

26. G. Bauer, J. Drobits, C. Fabjan, H. Mikosch, P. Schuster, J. Electroanal. Chem. 1997, 427, 123.

27. K.J. Cathro, K. Cedzynska, D. C. Constable, J. Power Sources, 1985, 16, 53.

28. P. M. Hoobin, K. J. Cathro and J. O. Niere, J. Appl. Electrochem., 1989, 19, 943.

29. J. Miller, Extended Linear Chain Compounds, 2013, 1, 212

30. SAINTPLUS, Bruker AXS Inc., Madison, Wisconsin, USA, 2003.

31. G.M. Sheldrick, SADABS, Program for Empirical Absorption Correction, University of Gottingen, Gottingen, Germany, 1996.

32. G.M. Sheldrick, Acta Crystallogr. 1998, 64A, 112. 